\title{
Informalisierung und Arbeitskämpfe in Indien. Eine zeithistorische Perspektive auf die Gegenwart
}

Die Arbeitskämpfe Indiens werden - trotz ihrer langen Tradition - in Europa selten wahrgenommen. Dies mag in der Annahme einer unüberbrückbaren kulturellen und historischen Andersartigkeit begründet sein. Die Konsequenzen der im Folgenden dargestellten fortschreitenden „Informalisierung“ von Arbeitsverhältnissen für die Dynamik von Arbeitskämpfen weisen jedoch deutlich über den indischen Fall hinaus. „Familienähnlichkeiten“ mit zeitgenössischen Prozessen in anderen, auch europäischen Teilen der Welt, sind unübersehbar.

RAVI AHUJA

\section{Europäische Gewerkschaften und indische Arbeitswelt}

Seit Ende des Zweiten Weltkrieges ist das internationale Interesse bundesdeutscher Gewerkschaften fast ausschließlich auf die nordatlantische Weltregion beschränkt geblieben. Arbeitswelt, Arbeitskonflikte und Gewerkschaften in Afrika, Asien oder Lateinamerika wurden hierzulande in der Regel nur im Rahmen entwicklungspolitischer Bestrebungen etwa zur „Professionalisierung“ von „Dritte-WeltGewerkschaften“ und zur Eindämmung kommunistischen Einflusses thematisiert. Kommunikation und koordiniertes Handeln auf gleicher Augenhöhe erschien aufgrund der vorherrschenden modernisierungstheoretischen Prämissen als wenig dringlich: In der Gegenwart Europas wurde die Zukunft der „Dritten Welt“ gesehen; langfristig wurde mit einer Ausdehnung des Wohlfahrtsstaates und von Strukturen innerbetrieblicher Demokratie nach europäischem Vorbild gerechnet. Wenn indische Arbeiter folglich in einer anderen Zeit lebten als ihre deutschen Kollegen, so gab es wenig Grundlagen für gemeinsames Handeln: Europäische Gewerkschaften mussten nur in ihren eigenen Ländern neue Standards setzen, der Rest der Welt würde - so die gängige Einschätzung - schon nachfolgen.

Die realgeschichtlichen Prozesse lassen den ideologischen Charakter dieser modernisierungstheoretischen Annahmen heute klarer hervortreten:
(1) Die wohlfahrtsstaatliche Regulierung der Arbeitswelt erweist sich als begrenztes, differenziertes, nur relativ stabiles, bisweilen sogar umkehrbares Phänomen. Dass sie quasi ein gesetztes Ziel universalgeschichtlicher Entwicklung sei, das in Europa nur schneller erreicht werde als anderswo, hat sich nicht bestätigt.

(2) Die zunehmende globale Verflechtung von Produktionsprozessen unterstreicht, dass etwa deutsche und indische Lohnabhängige durchaus in derselben Zeit leben. Sie konkurrieren nicht nur miteinander (Produktionsverlagerung), sondern sind auch ähnlichen Regulierungsdynamiken (Informalisierung) ausgesetzt (Mayer-Ahuja 2013).

(3) Zunehmende Verflechtung führt aber nicht zur Homogenisierung der Arbeitswelten oder der Lebens- und Arbeitsbedingungen: Transnationale Produktionsketten bleiben nur solange profitabel, wie ein Gefälle zwischen den Produktionskosten verschiedener Standorte fortbesteht. Beginnt sich die Schere zu schließen, wird in der Regel erneut verlagert. So wird durch Globalisierung Ungleichheit befestigt und reproduziert.

Die Fragen, wie auf den Trümmern modernisierungstheoretischer Ideologie Grundlagen für eine Kooperation von Gewerkschaften Europas mit denen des Südens neu bestimmt werden können und die Schützengrabenperspektive der Standortverteidigung überwunden werden 
kann, erscheinen mir völlig offen. Eine intensivere Beschäftigung mit andersartigen, aber verbundenen Arbeitswelten kann ein erster Schritt sein.

Der Beitrag gibt zunächst einen knappen Überblick über die historische Entwicklung der industriellen Arbeitswelt Indiens und ihre Zweiteilung in einen sogenannten formellen und informellen Sektor (Abschnitt 2 und 3). Damit wird der wesentliche Kontext, der für die Analyse sich wandelnder Arbeitskampfdynamik in Indien erforderlich ist, skizziert. Danach können wir uns den Transformationsprozessen seit Beginn der Liberalisierungspolitik (ca. 1991) und den Folgen für die Entwicklung von Arbeitskämpfen zuwenden (4). Schließlich werden vier Hypothesen zu den Implikationen von Informalisierungsprozessen für die Arbeitskampfdynamik des heutigen Indiens formuliert (5). Der Artikel argumentiert, dass Informalisierung in Indien die Wirksamkeit rechtsförmig regulierter Arbeitskämpfe zwar reduziert, die Bedeutung des heute nachlassend umgesetzten Arbeitsrechts für die Formulierung von Gerechtigkeitsnormen und Erwartungen der abhängig Beschäftigten gegenüber Staat und Unternehmen jedoch nicht untergraben hat. Die Erfahrung der Uneinlösbarkeit dieser Erwartungen in einer zunehmend informalisierten Arbeitswelt führt auch dazu, dass rechtlich normierte industrielle Konflikte in der Tendenz von scheinbar „primitiveren“, „,archaischen“ Arbeitskampfformen verdrängt werden, deren Brutalisierung und relative Unorganisiertheit sich jedoch vor dem Hintergrund zeitgenössischer Globalisierungsprozesse erklärt.

\section{Die Entwicklung der Industriearbeit in Indien}

\subsection{Industrialisierung}

Ein erster Industrialisierungsschub erfasste Indien im letzten Viertel des 19. Jahrhunderts, blieb jedoch zunächst stark auf die Textilindustrie und wenige lokale Schwerpunkte konzentriert: Bombay wurde zum Zentrum der Baumwollverarbeitung, der Großraum Kalkutta beherbergte fast die gesamte Juteindustrie. In den 1920er Jahren beschäftigten die Textilindustrien dieser beiden Metropolen allein rund eine halbe Million Menschen (Ahuja 2003). Zwischen und vor allem während der Weltkriege vollzog sich zugleich eine Erweiterung und Diversifizierung der industriellen Basis Indiens, wobei der Beschäftigungseffekt angesichts der Größe und nach wie vor weitgehend agrarischen Struktur des Landes begrenzt blieb: In den späten 1940er Jahren, als Indien unabhängig wurde, waren bei einer Bevölkerung von 400 Mio. lediglich 12 Mio. Menschen im weiterverarbeitenden Gewerbe beschäftigt und davon nur 2,6 Mio. in Fabriken bzw. größeren Betriebseinheiten (ILG 1946, S. 123).
Selbst dieser geringe Anteil wurde erst durch einen signifikanten Ausbau des öffentlichen Sektors und der Schwerindustrie im Rahmen der Kriegswirtschaft (1940 - 45) erzielt, ein Prozess, der im Zuge der postkolonialen Fünfahrespläne ab 1951 dann in erheblich gesteigertem Tempo fortgeführt wurde.

$\mathrm{Ab}$ den frühen 1980er Jahren war ein tief greifender industrieller Strukturwandel zu beobachten, dessen zunächst sichtbarster Ausdruck der Niedergang der im späten 19. Jahrhunderts entstandenen Industriehochburgen wie Bombay, Kalkutta, Ahmedabad oder Kanpur war (Breman 2004). Zugleich etablierten sich aber neue Industrien (u. a. Pharma-Industrie, Automobilzulieferer und IT). Die beschäftigungsintensive Textilindustrie wurde nicht abgebaut, sondern räumlich verlagert und flexibel reorganisiert (vgl. Abschnitt 2.3), während transnationale Konzerne nach der Liberalisierung der indischen Wirtschaft ab 1991 ihre Präsenz etwa im Fahrzeugbau und der metallurgischen Industrie massiv verstärkten. In Indien ist daher in den letzten drei Jahrzehnten kein eindeutiger Trend zur Deindustrialisierung zu beobachten, sondern eher, wie in China, eine Zweiteilung zwischen „Rostgürtel“ und „Sonnengürtel“: Auf der einen Seite setzt sich in alten Industrieschwerpunkten eine postindustrielle Dienstleistungsstruktur durch, die ihren sichtbarsten Ausdruck darin findet, dass Einkaufszentren und teure Wohnquartiere an die Stelle viktorianischer Fabriken treten. Auf der anderen Seite verzeichnen wir den Aufstieg neuer industrieller Konzentrationen, oft in der Peripherie urbaner Metropolen oder sogar in eher ländlichen Regionen. Dabei bleibt der Agrarsektor, in dem die Hälfte der inzwischen rund 500 Mio. Menschen zählenden Arbeitsbevölkerung Indiens tätig ist, für die Beschäftigungsstruktur von überragender Bedeutung (NCEUS 2009, S. 18 und passim). Formale Selbstständigkeit hat in der gesellschaftlichen Organisation der Arbeit weiterhin großes Gewicht, sodass Lohnarbeit in Indien erst zur Jahrtausendwende zur verbreitetsten Erwerbsform wurde.

\section{2 „Arbeiterfrage“ und Trennung des „infor- mellen Sektors“ vom „formellen Sektor“}

Wurde Industriearbeit also bereits im späten 19. Jahrhundert zu einer signifikanten sozialen Realität, so wurde die „Arbeiterfrage“ erst zwischen den Weltkriegen als zentrales politisches Problem anerkannt. Dies war zum Teil Ergebnis des Wachstums und der Diversifizierung der Industrie selbst, vor allem aber eine Reaktion auf eskalierende Arbeitskämpfe (vgl. Abschnitt 4). Hinzu kam, dass Geschäftsstrategien, die das Fixkapital minimierten und die daraus resultierenden Produktivitätseinbußen durch Niedrigstlöhne ausglichen, einer zunehmend spürbaren japanischen Konkurrenz unterlegen waren, die niedrige Löhne mit höherer Produktivität kombinierte. Die Regulierung industrieller Beziehungen, sozialpolitische Initiativen, die „Rationalisierung“ der Produktion und die „Planung“ nationaler Arbeitsressourcen und ihrer Reproduktion wurden vor 
diesem Hintergrund ab den 1920er Jahren zu wichtigen Themen der politischen Debatte, sowohl in kolonialen als auch in nationalistischen Kreisen Indiens (Ahuja 2003; 2013).

Die zunehmende Bedeutung der „Arbeiterfrage“ mag früheren Entwicklungen in Europa ähneln; zugleich lässt sich aber ein signifikanter Unterschied feststellen: Die rigorose Eingrenzung des Geltungsbereichs von Arbeits- und Sozialrecht auf eine kleine Minderheit der Erwerbsbevölkerung war prägend für die formative Periode (1930er bis 1950er Jahre) der gesetzlichen, formellen Regulierung der Arbeitswelt Indiens. Die gesetzliche Regulierung von Arbeitsbeziehungen resultierte also nicht in einem „Normalarbeitsverhältnis“, das zumindest für eine Mehrheit der Beschäftigten erfahrbar gewesen wäre, obwohl immer wieder in Aussicht gestellt wurde, den Geltungsbereich in Zukunft schrittweise auf die gesamte Arbeitswelt zu erweitern. Realiter wurde vielmehr eine Segmentierung des Arbeitsmarktes politisch erzeugt und fein kalibriert (Mohapatra 2005; Simeon 2005).

Zunächst wurde zwischen einem organisierten und einem unorganisierten Sektor differenziert; seit den 1970er Jahren setzte sich im offiziellen und wissenschaftlichen Sprachgebrauch dann weltweit die Unterscheidung zwischen einem formellen und informellen Sektor durch. Dem organisierten oder formellen Sektor werden in Indien als Kapitalgesellschaften eingetragene Firmen mit mehr als zehn Beschäftigten zugerechnet, in denen, zumindest der Theorie nach, das Arbeitsrecht (vor allem das Fabrikgesetz) und beschäftigungsabhängige Sozialleistungen Geltung haben (Breman 2013). Zum informellen Sektor, in dem das Arbeits- und Sozialrecht prinzipiell keine Gültigkeit hat, wird fast die gesamte Landwirtschaft (mit Ausnahme der Plantagen) gezählt, darüber hinaus aber nach offiziellen Angaben von 2009 auch knapp 74\% der nicht-agrarischen Arbeitsmarktbereiche (Industrie und Dienstleistungen).

Eine neue Definition von Informalität, die von einer indischen Regierungskommission (National Commission for Enterprises in the Unorganised Sector) im Jahre 2007 publiziert wurde, verdeutlicht die zunächst etwas verwirrend klingende Unterscheidung zwischen Beschäftigung im informellen bzw. formellen Sektor einerseits und informeller bzw. formeller Beschäftigung andererseits. Während nämlich 2009 ca. $86 \%$ der indischen Erwerbsbevölkerung einer Beschäftigung im informellen Sektor nachgingen, erfolgte faktisch rund $93 \%$ der Erwerbstätigkeit in Form informeller Beschäftigung. Diese Diskrepanz verweist auf einen wichtigen Prozess, nämlich den der zunehmenden Informalisierung innerhalb des formellen Sektors, vor allem in Form von Leiharbeit: Knapp die Hälfte der Beschäftigten des formellen Sektors sind inzwischen weder durch Arbeitsrecht geschützt noch durch beschäftigungsbezogene Sozialleistungen abgesichert, während der gesamte Beschäftigungszuwachs im „organisierten Sektor“ während des vergangenen Wachstumsjahrzehnts der indischen Volkswirtschaft im Bereich „informeller“ Beschäftigung erfolgte (NCEUS 2009, S. 12f. und passim).

\subsection{Fallbeispiel: Informalisierung der Textil- industrie}

Was sich hinter den enormen quantitativen Ausmaßen des informellen Sektors verbirgt, lässt sich am besten an einem Fallbeispiel erläutern. Die baumwollverarbeitende Textilindustrie ist einer der ältesten Zweige indischer Fabrikproduktion. Obwohl hoch spezialisierte Handweberei in Indien nie verschwunden ist und darüber hinaus ein beträchtlicher Teil der Textilproduktion seit der ersten Hälfte des 20. Jahrhunderts mit elektrifizierten Webmaschinen in Kleinbetrieben (sogenannte Powerlooms) erfolgt, war die Fabrikproduktion von Baumwolltextilien bis in die 1980er Jahre ein wichtiger Beschäftigungssektor: allein in Bombay arbeiteten damals rund 250.000 Menschen in Textilfabriken (Weersch 1992).

Seitdem ist ein dramatischer Niedergang zu verzeichnen: Heute werden nur noch $5 \%$ der indischen Textilien in formell regulierten Fabriken produziert. Beherrschend ist heute der Powerloom-Sektor, in dessen (juristisch oder real) meist kleinen Betriebseinheiten das Arbeitsrecht keine Gültigkeit hat. In mehr als 500.000 solcher Betriebseinheiten erfolgt über $60 \%$ der indischen Textilproduktion mit rund 2 Mio. Webmaschinen und 4-5 Mio. Beschäftigten (Government 2006, Kap. 2.2; 2012; Anjum/Thakor 2011). Der informelle Sektor ist hier (wie in anderen Industrien) kein Restbestand einer vormodernen Arbeitswelt, sondern in großen Teilen das Ergebnis jüngerer industrieller Umstrukturierung mit dem Ziel der Kostensenkung und Flexibilisierung. Die Verlagerung der Textilproduktion von formell regulierten Fabriken in neue Konzentrationen von kleinen Betriebseinheiten ging mit Lohnverlusten von bis zu $50 \%$, mit Ortsverlagerungen und mit dem weitgehenden Austausch von Belegschaften einher: Industrielle Stammbelegschaften wurden großenteils durch gewerkschaftlich unorganisierte Migranten ersetzt, die häufig zwischen den neuen Textilzentren und den bis zu $1.500 \mathrm{~km}$ entfernten Heimatdörfern zirkulieren, in denen ihre Familien leben.

Die neuen Konzentrationen der Textilproduktion sind oft durchaus massiv: So beherbergten Bhiwandi, in der Peripherie Bombays gelegen, und Surat im westindischen Bundesstaat Gudscharat nach offiziellen Zahlen von 2008 Powerloom-Konzentrationen von 340.000 bzw. 450.000 Webmaschinen; nach inoffiziellen Schätzungen stehen in diesen beiden Orten deutlich mehr, nämlich rund die Hälfte der indischen Powerlooms (Government 2006, Kap. 2.2). Die Produktion erfolgt durchaus auch in großen Fabrikhallen, in denen aber formal zahlreiche, juristisch unabhängige Unternehmen tätig sind. Dennoch sind diese neuen industriellen Konzentrationen gesellschaftlich weit weniger sichtbar als ihre Vorgänger: Während im frühen 20. Jahrhundert bedeutende urbane Zentren wie Bombay oder Ahmedabad als Manchester Indiens bezeichnet wurden, schmücken sich heute eher unbekannte Orte mit diesem zweifelhaften Ehrentitel. Klassische Industriearbeit ist in der Textilbranche also keineswegs verschwunden; die neuen Sweatshops 
sind lediglich in eine von der Öffentlichkeit gewöhnlich ignorierte Schattenwelt verbannt worden.

\section{Politische Regulierung industrieller Beziehungen}

Auf dem Gebiet der industriellen Beziehungen wurde von der Regierung des postkolonialen Indiens nach $1947 \mathrm{im}$ Wesentlichen die Politik des britischen Kolonialregimes fortgeführt: Das Streikrecht wurde eingeschränkt und in staatswichtigen Branchen ganz ausgesetzt; „repräsentative“, also mit Verhandlungsmandat versehene Gewerkschaften mussten von staatlichen Stellen anerkannt werden; Arbeitskonflikte unterlagen staatlicher Zwangsschlichtung. Im Ergebnis entstand ein Regime industrieller Beziehungen, das von Tarifautonomie nicht weiter entfernt hätte sein können und vielleicht als staatszentrierter Tripartismus bezeichnet werden kann.

In dem Maße, wie der Zugang zu staatlichen Regulierungsinstanzen und Regierungskorridoren zum Schlüssel für erfolgreiche Interessenvertretung wurde, entfaltete sich nun innerhalb der Gewerkschaften ein Prozess der Bürokratisierung, wobei die Bindung an politische Parteien enger und ausschließlicher wurde. Zugleich engte sich gewerkschaftliche Arbeit zunehmend auf die so institutionalisierten Aushandlungswege und damit auf den formellen Sektor ein: In der informellen Ökonomie blieben ja nur die Formen sozialer Mobilisierung, denen „repräsentative“ Gewerkschaften abschwören sollten (Hensman 2011).

Seit Indira Gandhis Notstandsregierung (1975 - 77) war dann ein Niedergang des staatszentrierten Tripartismus, dieses spezifischen Typs korporatistischer Regulierung, zu verzeichnen. Mit politischer Rückendeckung aus den Regierungsetagen wurden im Laufe eines Jahrzehnts die gewerkschaftlichen Hochburgen Indiens geschliffen. Der in einer verheerenden Niederlage und der vollständigen Verlagerung und Umstrukturierung der Industrie endende Bombayer Textilarbeiterstreik von 1982-84, eine der wohl bittersten Streikbewegungen der Geschichte mit ca. 200.000 Teilnehmern, markierte den Höhepunkt dieser Entwicklung (Weersch 1992). Nachdem 1991 die bis dahin schleichende neoliberale Wende in der Wirtschafts- und Sozialpolitik öffentlich erklärt und verschärft wurde, zeigten sich die Elemente des Regimewechsels bezüglich der Regulierung der Arbeitswelt deutlich:

(1) Die Empörung über die „Privilegien“ der formell beschäftigten „Arbeiteraristokratie“ gehörte nun zum guten Ton in Politiker-, Medien- und Wissenschaftsdiskursen.

(2) Die indischen Bundesstaaten begaben sich miteinander in einen Unterbietungswettbewerb zur Bereitstellung des „besten Geschäftsklimas“ einschließlich der Einrichtung von wirtschaftlichen Sonderzonen, in denen das Arbeitsrecht keine Gültigkeit besitzt, und der massiven polizeilichen Unterbindung von Arbeitskämpfen.

(3) Das bestehende Arbeitsrecht wurde zwar als Arbeitsmarktrigidität kritisiert, aber nicht auf dem Wege der Gesetzgebung außer Kraft gesetzt. Stattdessen vollzog sich, was treffend als "Arbeitsmarktreform unter der Tarnkappe“ bezeichnet worden ist (Shyam Sundar 2010): Geltendes Arbeitsrecht wird mit richterlicher und politischer Unterstützung häufig nicht implementiert und steht so den Beschäftigten im Konfliktfall oft nicht mehr als Ressource zur Verfügung.

(4) Auf verschiedenen Wegen ist ein Prozess der Informalisierung, also der Umwandlung formeller Beschäftigungsverhältnisse in informelle, eingeleitet worden. Dieser Prozess kann durch Verlagerung aus dem formellen in den informellen Sektor (etwa durch Betriebsaufteilung, räumliche Verlagerung und Dezentralisierung) oder durch innere Aushöhlung des formellen Sektors (typischerweise Leiharbeit) erfolgen. Diese oft nicht rechtskonforme Aushöhlung des formellen Beschäftigungsstatus ist in privatwirtschaftlichen Betrieben leichter durchsetzbar als in Staatsbetrieben, weshalb Privatisierung hier in manchen Fällen einen Einstieg bot (Breman 2013).

(5) Schließlich führte der Regimewandel dazu, dass die Gewerkschaften ihren Einfluss auf die Politik der Parteien weitgehend verloren und Probleme der Arbeitswelt nur noch geringes öffentliches Echo erzielen konnten. Mit der Schwächung der Gewerkschaftsbünde einher ging die Entstehung zahlreicher „unpolitischer“, auf Partikularinteressen bestimmter Beschäftigungsgruppen fokussierter Betriebsgewerkschaften, parteiunabhängiger militanter Gewerkschaften, nach Kastenzugehörigkeit oder Ethnizität organisierter identitärer Zusammenschlüsse abhängig Beschäftigter sowie verschiedener „wohltätiger" Nichtregierungsorganisationen (NGO), die bisweilen klassische Gewerkschaftsfunktionen übernahmen. Die etablierten Gewerkschaften richten sich nur allmählich auf die veränderte Situation ein, indem sie sich für informell Beschäftigte stärker öffnen (Bhattacharjee/ Ackers 2010; Hensman 2011).

\section{Indische Arbeitskämpfe}

Diese langfristigen Tendenzen in der Politik industrieller Beziehungen können nur im Kontext der Geschichte indischer Arbeitskämpfe verstanden werden. So war die Genese des oben beschriebenen staatszentrierten Tripartismus in den mittleren Dekaden des 20. Jahrhunderts in vieler Hinsicht eine Reaktion auf den Anstieg von Streik- 
bewegungen. Wenn Beverly Silvers quantitative Langzeitanalyse eine weltweite Verstärkung von Streikbewegungen nach beiden Weltkriegen (und nach 1945 insbesondere außerhalb der atlantischen Weltregion) festgestellt hat, so gilt das in vollem Umfang auch für den indischen Fall (Silver 2003). Eine bis dahin unbekannte Häufigkeit von Arbeitskämpfen in den Jahren 1919-21 beförderte die Gründung zahlreicher Gewerkschaften; in den späten 1920er Jahren und erneut in den späten 1930er Jahren kam es nicht nur zu weiteren Streikwellen, sondern auch zu einer größeren Militanz und Intensität der Arbeitskämpfe, die mit einer politischen Radikalisierung der Arbeiterbewegung einhergingen und die Regulierung von Arbeitskämpfen zu einem zentralen politischen Thema machten. Die allgemeine politische und soziale Krise nach Ende des Zweiten Weltkrieges brachte neben großflächigen Bauernaufständen, pogromartigen Zusammenstößen zwischen Hindus und Muslimen und einer gewachsenen Unruhe innerhalb der Streitkräfte des sich zurückziehenden Kolonialstaats auch eine ungekannte Verbreiterung von Streikbewegungen hervor, die in hohem Maße auch den öffentlichen Dienst einbezogen. In der abziehenden kolonialen wie auch in der sich formierenden postkolonialen Regierung verfestigte sich der Eindruck, am Kraterrand eines ausbrechenden Vulkans zu sitzen (Sarkar 1985, Kap. 8). Die Pflöcke eines rigiden staatszentrierten Tripartismus wurden nun, vor allem in den Jahren 1947 und 1948, in großer Eile eingeschlagen.

Wie bereits argumentiert, trug dieser staatszentrierte Tripartismus zu einer Ausdifferenzierung zwischen einer formellen (rechtsförmig regulierten) und einer informellen (vor allem durch soziale Mechanismen regulierten) Ökonomie sowie zu einer gewissen Abwendung der Gewerkschaften von der Interessenvertretung der informell Beschäftigten bei. Eine Eindämmung von Streikbewegungen konnte bis Mitte der 1970er Jahre allerdings nicht durchgesetzt werden: die Zahl der statistisch erfassten Arbeitskämpfe erreichte, im Gegenteil, immer neue Höhen. Erst als autoritäre Tendenzen in der postkolonialstaatlichen Entwicklung 1975 in der Ausrufung des nationalen Notstands kulminierten und Premierministerin Indira Gandhi zwei Jahre lang den demokratischen Prozess weitgehend aussetzte, kam es zu einer statistisch gut nachvollziehbaren Wende (Abbildung 1). Dies ging einher mit der allmählichen Einleitung einer wirtschafts- und sozialpolitischen Wende. Die Häufigkeit statistisch erfasster Arbeitskämpfe fiel nun deutlich ab, wobei Aussperrungen (hinter denen sich auch versteckte Betriebsschließungen unter Umgehung des Kündigungsschutzes verbergen können) gegenüber Streiks stetig an Bedeutung zunahmen (Shyam Sundar 2010).

Dabei ist zu beachten, dass die offiziellen Arbeitskampfstatistiken nur bedingt aussagekräftig sind. Die Meldung von Streiks an die zuständigen offiziellen Stellen ist nicht verpflichtend; die Arbeitsverwaltungen, denen auf der lokalen Ebene die Erfassung obliegt, waren vor 1955 administrativ schwach aufgestellt und haben seit der offiziellen
ABB.

\section{Registrierte Arbeitskämpfe in Indien 1921 - 2010}

Angaben in absoluten Zahlen

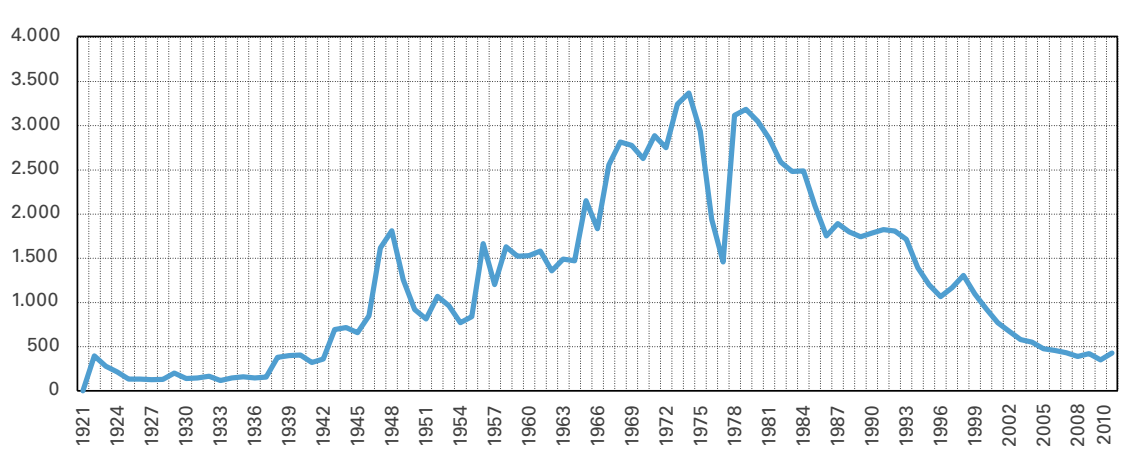

Quelle: Darstellung und Zusammenstellung des Autors aus seit 1921 von der staatlichen Arbeitsverwaltung veröffentlichten Arbeitskampfstatistiken.

Wende zur wirtschaftspolitischen Liberalisierung im Jahre 1991 nachlassende Priorität; die indischen Bundesstaaten buhlen um die Gunst nationaler und internationaler Investoren, indem sie ihr besonders vorteilhaftes Geschäftsklima propagieren, für das eine geringe Streikfrequenz ein wichtiger Indikator ist; Streiks im öffentlichen Dienst sind 2003 untersagt worden, während politische Streiks und andere Ausstände, die nicht den Normen des Arbeitskampfgesetzes entsprechen, nicht erfasst werden (Shyam Sundar 2010). Dass wir es mit einer erheblichen, von den offiziellen Statistiken nicht erfassten Frequenz von unregulierten, „wilden“ Arbeitskämpfen zu tun haben, wird auch darin deutlich, dass seitens der Unternehmerverbände (insbesondere der Föderation der Indischen Industrie- und Handelskammern) die Gesamtentwicklung der industriellen Beziehungen keineswegs als positiv eingeschätzt und vielmehr eine besorgniserregende Zunahme gewaltsamer Arbeitskonflikte beklagt wird (Ahuja 2013). Abbildung 1 zur Entwicklung indischer Arbeitskämpfe seit 1921 übertreibt daher mit großer Sicherheit die Tiefstände vor 1955 und nach 1991. Zuverlässige Auskunft gibt es eher über die Konjunkturen indischer Politik der industriellen Beziehungen und über den Aufstieg und Fall von „Arbeit“ als öffentlicher, politischer Kategorie als über die Häufigkeit und Dynamik von Arbeitskämpfen.

\section{Informalisierung und Arbeitskämpfe im zeitgenössischen Indien}

Aus der hier nur in Grundzügen angedeuteten zeithistorischen Analyse ergeben sich vier Hypothesen über den Zusammenhang von Prozessen der „Informalisie- 
rung" und den neuen Dynamiken in indischen Arbeitskämpfen. Sie bedürfen sämtlich wissenschaftlicher Überprüfung durch weitere Forschungen und gehen bewusst an die Grenze des derzeitigen Kenntnisstands. Zudem tragen die diagnostizierten, widerstreitenden Tendenzen partiell sicherlich den Besonderheiten des indischen Falls Rechnung, könnten in anderen Teilen aber auf breitere, für andere Teile der Welt ebenfalls relevante Entwicklungen verweisen. Der vorliegende Artikel kann eine solche, systematischen Vergleich erfordernde Unterscheidung nicht leisten, soll aber zu weiteren komparativen Forschungen ermutigen.

\subsection{Informalisierung, nicht Deregulierung}

Hypothese 1: Informalisierung darf nicht mit dem Ende arbeitsmarktrelevanter sozialpolitischer Regulierung (Deregulierung) verwechselt werden, sondern impliziert lediglich einen Politikwechsel, der die direkte rechtsförmige Regulierung des Arbeitsverhältnisses (vor allem durch Arbeitsrecht) und damit auch die Wirksamkeit rechtskonformer, regulärer Arbeitskämpfe reduziert.

So ist in Indien etwa die bereits erwähnte nachlassende Durchsetzung und Einklagbarkeit des bestehenden Arbeitsrechts mit neuen, durchaus signifikanten und arbeitsmarktrelevanten sozialpolitischen Initiativen einhergegangen. Diese sozialpolitischen Maßnahmen lassen sich grob in zwei Kategorien unterteilen. Zum einen geht es hier um Maßnahmen der positiven Diskriminierung zugunsten vor allem unterer sozialer Statusgruppen (Kasten), etwa die Quotierung von Stellenbesetzungen im öffentlichen Dienst. Zum anderen handelt es sich um allgemeine Beschäftigungsprogramme, insbesondere den National Rural Employment Guarantee Act (NREGA) von 2005, der abhängig Beschäftigten im ländlichen Indien mit Hilfe staatlicher Infrastrukturprogramme eine jährliche Mindestbeschäftigung von 100 Tagen garantiert. Beide Kategorien haben eindeutig beschäftigungs- und damit arbeitspolitischen Charakter. Beide beziehen sich aber auf Rechtsverhältnisse und Rechtsansprüche zwischen Staatsbürgern (bzw. bestimmten benachteiligten Gruppen von Staatsbürgern) und dem Staat, während die Rechtsverhältnisse und Rechtsansprüche zwischen Unternehmern und Beschäftigten (Arbeitsverhältnisse im engeren Sinn) nicht Gegenstand dieser sozialpolitischen Programme sind. Wenn also die Quotierung nach Kastenzugehörigkeit oder die Finanzierung und Umsetzung der ländlichen Mindestbeschäftigungsgarantie zum Gegenstand teilweise heftiger sozialer und politischer Konflikte werden, bleiben die Unternehmen weitgehend außerhalb der Schusslinie. Umgekehrt hat damit der regulierte, durch gesetzliche Regelung industrieller Beziehungen normierte und an feste Verhandlungsroutinen und Schlichtungsprozeduren gebundene Arbeitskampf als rechtsbasierte Form sozialer Interessendurchsetzung seitens der abhängig Beschäftigten an Gewicht verloren.

\subsection{Arbeitsrecht als Erwartungshorizont}

Hypothese 2: Rechtsförmige Regelungen des Arbeitsverhältnisses definieren, trotz des extrem eingeschränkten Geltungsbereichs und der nachlassenden Einklagbarkeit des Arbeitsrechts, nach wie vor die Erwartungshorizonte abhängig Beschäftigter.

Für die formelle Ökonomie erkämpfte Errungenschaften wie Kündigungsschutz oder Pensionsanspruch gehören also nicht allein der sogenannten Arbeiteraristokratie, auch wenn besser gesicherte Beschäftigte immer wieder versucht haben, sich gegen die Billigkonkurrenz auf dem Arbeitsmarkt einzuigeln, also Closed Shops einzurichten, und obwohl Solidarisierung zwischen formell und informell Beschäftigten innerhalb der Betriebe (also etwa zwischen regulär Beschäftigten und Leiharbeitern) schwer zu erzielen ist. Dennoch bestimmen diese Errungenschaften trotz zunehmender Aushöhlung immer noch die Erwartungshorizonte zumindest von Teilen der abhängig Beschäftigten bis weit in die informelle Ökonomie hinein. Insbesondere liefern sie ein Vokabular und eine Grammatik, mit deren Hilfe konkrete Forderungen in Arbeitskämpfen formuliert werden können, und ein Instrument zur Legitimierung dieser Forderungen.

Zwei Beispiele, je eines aus der informellen und der formellen Ökonomie, mögen dies illustrieren. Zum einen wurden im informell organisierten Textilsektor, der Powerloom-Industrie, im Jahre 2013 anlässlich verschiedener Arbeitskämpfe Forderungen erhoben, welche im Kern die Anwendung von Arbeitsschutzregelungen und beschäftigungsbedingten Sozialleistungen beinhalteten, die bisher Beschäftigten des formellen Sektors vorbehalten sind: So wurde in Ichalkaranji (im westindischen Bundesstaat Maharashtra) erfolgreich der Achtstundentag (The Hindu, 3. März 2013), im südindischen Rajapalayam sogar die Einbeziehung in Pensionsfonds und Sozialversicherungsschutz im Rahmen des Employees' State Insurance Scheme (ESI) gefordert (The Hindu, 6. Juni 2013). Zum anderen ist auch unter den segmentierten Belegschaften der formellen Ökonomie erkennbar, dass als allgemeingültig verstandene „Gerechtigkeitsstandards“ bzw. soziale Rechte aus dem im formellen Sektor gültigen Arbeitsrecht abgeleitet werden. Im Maruti-Automobilwerk des Honda-Konzerns in Manesar, unweit von Delhi, gelang etwa im Ergebnis mehrjähriger Auseinandersetzungen im Jahre 2011 eine Solidarisierung der Stammbelegschaft mit der als Leiharbeiter beschäftigten anderen Belegschaftshälfte, wobei eine Regularisierung der Leiharbeitsverhältnisse nach dem Arbeitsrecht des formellen Sektors und unter Einhaltung des (in vielen Betrieben regelmäßig unterlaufenen) Leiharbeitsgesetzes von 1970 gefordert wurde (Singh/Sawhney 2011).

\subsection{Brutalisierung der Arbeitsbeziehungen}

Hypothese 3: Die nachlassende staatliche Regulierung oder „Privatisierung“ des Arbeitsverhältnisses fördert eine Bru- 
talisierung der alltäglichen Arbeitsbeziehungen wie auch der Arbeitskämpfe.

Informalisierung innerhalb und außerhalb des formellen Sektors erzeugt unbeherrschbare industrielle Gewaltspiralen. Körperliche Gewalt und psychische Misshandlung (etwa in Form demütigender Beleidigungen) werden auch in Konzernbetrieben häufiger zur alltäglichen Disziplinierung von Beschäftigten eingesetzt. Streikende sehen sich zudem mit massiven polizeilichen Übergriffen konfrontiert, in denen verfassungsmäßige Individualrechte nicht selten ausgesetzt werden. Umgekehrt kann sich die Frustration über die faktische Uneinklagbarkeit von formell garantierten Rechten in spontanen, gewaltsamen Konfrontationen entladen. Dies ist in den vergangenen Jahren besonders in Betrieben beobachtet worden, wo formell abgesicherte Beschäftigte mit Leiharbeitern Seite an Seite für unterschiedlichen Lohn und mit abweichenden Rechten beschäftigt sind.

Zwei spektakuläre Fälle im Jahre 2012 setzten in Indien eine öffentliche Debatte zu dieser Problematik in Gang. Der erste ereignete sich im Januar 2012 bei Regency Ceramics im südindischen Yanam, einem der größten Fliesenhersteller des Landes, der zweite im Juli 2012 im bereits erwähnten Maruti-Werk in Manesar. In beiden Fällen wurde im Zuge von Arbeitskonflikten massiv Gewalt gegen die Belegschaft eingesetzt (in Yanam durch die Polizei; für Manesar wurde das Anheuern betriebsfremder Schläger berichtet), was bei Regency Ceramics mit dem Tod eines lokalen Gewerkschaftsführers endete. Die nachfolgende Gewalteskalation führte in beiden Fällen zu schwerer Beschädigung der Produktionsanlagen sowie zum Tod des Betriebsleiters bzw. Personalchefs (Presseberichten zufolge im ersten Fall vorsätzlich, im zweiten als evtl. unbeabsichtigtes Ergebnis von Brandstiftung). In beiden Fällen waren langwierige, mit legalen Mitteln geführte Kämpfe zur Durchsetzung des gesetzlich verbrieften Rechts auf gewerkschaftliche Organisationsfreiheit gescheitert und hatten sich weitere gesetzlich verankerte Rechte (insbesondere das Verbot von Leiharbeit für reguläre Tätigkeiten) als uneinklagbar erwiesen. In der Folge eskalierten die Konflikte, übernahmen gewaltbereite Akteure die Initiative, wofür die Belegschaften und ihre Familien teuer, oft mit dem Verlust ihrer Existenzgrundlagen bezahlten. Zugleich hat die Brutalisierungsdynamik das Problem geregelter industrieller Beziehungen und betrieblicher Partnerschaft wieder auf die Tagesordnung indischer Unternehmerverbände gesetzt und Debatten über die Notwendigkeit einer strategischen Nachjustierung ausgelöst (Ahuja 2013; Sehgal 2012).

\subsection{Grenzen der Informalisierung}

Hypothese 4: In der informellen Ökonomie verweisen Arbeitskämpfe, oft in Formen jenseits des klassischen Streiks, auf die Grenzen der Informalisierung.

Informalisierungsprozesse, etwa im oben knapp umrissenen Fall der Textilindustrie, sind Teil von Unternehmens- strategien, die zum einen die zunehmend in transnationalen Wertschöpfungsketten organisierte Produktion flexibilisieren und Nachfrageschwankungen auf dem Weltmarkt kompensieren, zum anderen aber im Rahmen des internationalen Unterbietungswettbewerbs die Lohnkosten minimieren sollen. In der Powerloom-Industrie Indiens sind die Arbeitsverhältnisse entsprechend unsicher und jederzeit kündbar, während trotz starker Inflation versucht wird, die Löhne auf häufig 5.000 Rupien (also rund $€ 60$ Euro) im Monat einzufrieren. Dennoch klagen die Unternehmen verstärkt über den Preisdruck, der vor allem aus China und Bangladesh auf den indischen Textilsektor ausgeübt werde (Arun 2013). Die Strategie der indischen Textilunternehmen ähnelt verblüffend der ihrer Vorgänger in den 1930er Jahren, indem sie geringe Investitionen mit Niedriglöhnen kombinieren und die resultierenden Produktivitätseinbußen mit höherer Flexibilität auszugleichen versuchen. Das wird etwa darin deutlich, dass 2008 rund $75 \%$ der elektrischen Webmaschinen älter als 15 Jahre gewesen sein sollen (Government 2008, Kap. 2.2). Diese Rückkehr zu einem alten Geschäftsmodell stößt jedoch an ihre Grenzen, die in saisonalen und chronischen Personalengpässen sichtbar werden. Die Widersprüche resultieren auch in neuen Arbeitskämpfen im informellen Sektor, an denen alte wie neue Gewerkschaften nicht unbedingt beteiligt sind. Eine erhebliche Rolle spielen Formen unsichtbarer Organisation, also von Streikführungen, die nicht offen auftreten, um angesichts eines real nicht einklagbaren Streikrechts der Disziplinierung zu entgehen. Eine bedeutende Rolle spielen aus demselben Grund auch NGOs oder identitäre Zusammenschlüsse, in denen vor ökonomischen und polizeilichen Repressalien besser geschützte Honoratioren die Forderungen der Beschäftigten formulieren. Die Grenzen des Arbeitskampfes zur schlichten Abwanderung von Arbeitskräften sind auf dem Hintergrund irregulärer, jederzeit kündbarer Arbeitsverhältnisse oft fließend, wie das folgende Fallbeispiel zeigt:

Anfang 2011 ereignete sich in einem der größten Schwerpunkte der indischen Powerloom-Industrie, der Millionenstadt Surat im Bundesstaat Gudscharat, ein Arbeitskampf um höhere Löhne, der zeitweise 450.000 elektrifizierte Webstühle lahmgelegt haben soll. Der Arbeitskampf, von der offiziellen Arbeitsverwaltung zunächst nicht als Streik anerkannt, erfolgte ohne sichtbare gewerkschaftliche Organisation. Zahlreiche Beschäftigte der lokalen Textilindustrie stammen aus dem geografisch über $1.500 \mathrm{~km}$ und kulturell-sprachlich ebenfalls weit entfernten Bundesstaat Odisha. So erklärt sich, dass eine Art landsmannschaftlicher Organisation sich zum Sprachrohr der Streikenden aufschwang. Obwohl der Streik nach einigen Tagen offiziell endete, ergab eine Untersuchung, dass bis zu 40.000 streikende Powerloom-Arbeiter in ihre Heimatregion Odisha zurückgekehrt waren, dass zahlreiche weitere Migranten aus dem Osten Indiens in ihren Heimatdörfern dank der oben erwähnten ländlichen Mindestbeschäftigungsgarantie (NREGA) besser bezahlte Arbeit gefunden hatten und eine große Gruppe qualifizier- 
ter Weber in Surat selbst in die ebenfalls umfangreiche Stickereiindustrie gewechselt war (Kulkarni 2011).

\section{Schluss}

Der Beitrag hat vor dem historischen Hintergrund der Entwicklung industrieller Arbeitsverhältnisse und Beziehungen in Indien die Ergründung des Zusammenhangs von Informalisierung und dem Wandel der Arbeitskampfformen zum Gegenstand. Dabei wird beispielhaft auf zwei Teilindustrien, die Fahrzeugindustrie und die Powerloom-Industrie, zurückgegriffen. Erstere steht stellvertretend für die innere Front der Informalisierung, d.h. Informalisierung innerhalb des formellen Sektors vor allem durch Einsatz arbeitsrechtlich ungeschützter Leiharbeit. Die zweite steht dagegen für die äußere Frontlinie der Informalisierung, die vor allem durch Dezentralisierung zuvor formell regulierter Industrien oder durch Privatisierung und Einrichtung vom Arbeitsrecht ausgenommener Sonderwirtschaftszonen gezogen wird.

Die Ähnlichkeiten des hier skizzierten Doppelprozesses der indischen Informalisierung mit hierzulande beobachteten Prozessen sind unverkennbar: Auch hier wird selbst innerhalb der Hochburgen des Normalarbeitsverhältnisses, etwa durch verstärkten Einsatz von Leiharbeit in Großbetrieben, prekarisiert. Auch hier wird außerhalb dieser Hochburgen etwa durch staatliche Duldung „schwarzer “ Arbeitsmärkte (u. a. für häusliche Dienstleistungen) Informalität in der Arbeitswelt erzeugt. Die Ähnlichkeiten dürfen jedoch nicht übertrieben werden. Das enorme quantitative Übergewicht der informellen Ökonomie Indiens als Beschäftigungssektor für über $90 \%$ der Erwerbsbevölkerung schafft nicht nur im Vergleich zu Europa, sondern auch etwa zu Lateinamerika, grundlegend andere Bedingungen für die Führung von Arbeitskämpfen und die Gestaltung industrieller Beziehungen.

Zum Zeitpunkt der Abfassung dieses Artikels sind zudem auch die politischen Rahmenbedingungen für die Artikulation industrieller Konflikte in Indien im Wandel begriffen: Die Fortsetzung der seit zwei Legislaturperioden verfolgten Politik einer zumindest bruchstückhaften sozialpolitischen Abfederung des neoliberalen Transformationsprozesses scheint weder von der derzeit regierenden Koalition noch von den erstarkten Oppositionskräften angestrebt zu werden. Entsprechend ist nach den Parlamentswahlen im Mai 2014 mit einer verstärkt repressiven Politik gegenüber Streikbewegungen und einer fortwährenden Brutalisierung von Arbeitskämpfen zu rechnen. Die sozialen und wirtschaftlichen Grenzen der Informalisierung werden auch durch diese für alle Seiten gefährlichen Konflikte in Sichtweite bleiben.

\section{LITERATUR}

Ahuja, R. (2003): Arbeit und Kolonialherrschaft im neuzeitlichen Südasien: Eine Einführung, in: Preisendanz, K./Rothermund, D. (Hrsg.): Südasien in der "Neuzeit." Geschichte und Gesellschaft 1500-2000, Wien, S. 194-211 Ahuja, R. (2013): Das Ähnliche speist den Unterschied: Die globale Wohlfahrtsdebatte und die Erzeugung „informeller Arbeit" im Indien des 20. Jahrhunderts, in: Burchardt, H-J./Tittor, A./Weinmann, N. (Hrsg.): Arbeitspolitiken in globaler Perspektive: Informalität und Prekarisierung als Herausforderung,

Frankfurt a. M./New York, S. 123-144

Anjum, A./Thakor, D. V. (2011): An analytical study of the functioning and the problems of the powerloom industry in Maharashtra with special reference to Malegaon District, Nashik, in: International Journal of Trade, Economics and Finance 2 (3), S. 194-199

Arun, M.G. (2013): Looms in a tailspin, India today, 3. April Bhattacharjee, D./Ackers, P. (2010): Introduction: employment relations in India - old narratives and new perspectives, in: Industrial Relations Journal 41 (2), S. $104-121$

Breman, J. (2004): The making and unmaking of an industrial working class. Sliding down the labour hierarchy in Ahmedabad, India, Oxford

Breman, J. (2013): At work in the informal economy in India. A perspective from the bottom up, New Delhi, Oxford

Government of India, Ministry of Textiles, Office of the Textile Commissioner (2006): Handbook on powerloom, Mumbai, http://www.txcindia.gov.in/html/ PLcover.htm

Government of India, Ministry of Textiles, Office of the Textile Commissioner (2012): Official Indian Textile Statistics 2011-12, Mumbai, http://www.txcindia.gov. in/html/comp\%20table\%20pdf\%202011-12/officialindiantextile2011-12sub.htm

Hensman, R. (2011): Workers, unions, and global capitalism: lessons from India, Columbia University Press, New York

Indian Labour Gazette (ILG): 4,4 (1946)

Kulkarni, V.V. (2011): Mass exodus of workers: A case of strike in power loom industry in Surat, http://papers.ssrn.com/sol3/papers.cfm?abstract_id=1857405 Mayer-Ahuja, N. (2012): Arbeit, Unsicherheit, Informalität, in: Dörre, K./Sau-

er, D.Mittke, V. (Hrsg.): Kapitalismustheorie und Arbeit. Neue Ansätze soziologischer Kritik, Frankfurt a. M./New York, S. 289-301

Mohapatra, P. P. (2005): Regulated informality: Legal constructions of labour relations in colonial India, 1814-1926, in: Bhattacharya, S./Lucassen, J. (Hrsg.): Workers in the informal sector: studies in labour history, 1800-2000, New Delhi, S. 66-95

Mohapatra, P. P. (2013): A moving target: The worker in the mirror of law in India (unveröfftlichtes Manuskript)

National Commission for Enterprises in the Unorganised Sector (NCEUS)

(2009): The challenge of employment in India. An informal economy perspective, Bd. I, New Delhi, http://nceuis.nic.in/The_Challenge_of_Employment_in_India.pdf Rao, B. S. (1985): Some methodological aspects of strike statistics, in: Indian Journal of Industrial Relations 20 (4), S. 481-490

Sarkar, S. (1985): A critique of colonial India, Calcutta

Sehgal, R. (2012): "Maruti workers are the villains": Truth or prejudice?,

in: Economic and Political Weekly 47 (31), S. 12-15

Silver, B. (2003): Forces of labor. Workers' movements and globalization since 1870, Cambridge

Singh, J./Sawhney, N. (2011): Maruti workers speak about their strike, in: Economic and Political Weekly 46 (33), S. 12-13

Simeon, D. (2005): Calibrated indifference: Understanding the structure of informal labour in India, in: S. Bhattacharya/Lucassen, J. (Hrsg.): Workers in the informal sector. Studies in labour history, 1800-2000, New Delhi, S. 97-120

Shyam Sundar, K. R. (2010): Emerging trends in employment relations in India, in: Indian Journal of Industrial Relations 45 (4), S. 585-595

Weersch, H. W. M. (1992): The Bombay textile strike, 1982-83, Oxford

\section{AUTOR}

RAVI AHUJA, Prof. Dr., lehrt Moderne Indische Geschichte am Centre for Modern Indian Studies der Georg-August-Universität Göttingen. Arbeitsschwerpunkte: Probleme der Wirtschafts- und Sozialgeschichte Indiens, Globalgeschichte der Arbeit.

ravi.ahuja@phil.uni-goettingen.de 